\title{
Validity of venous phase delay assessment in balloon occlusion test of internal carotid artery
}

\author{
ZHU CHEN, LI-NAN HU, HUI ZHU and EN-HUA XIAO
}

Department of Radiology, The Second Xiangya Hospital of Central South University, Changsha, Hunan 410011, P.R. China

Received May 11, 2018; Accepted September 29, 2018

DOI: $10.3892 /$ etm.2018.7009

\begin{abstract}
The aim of the present study was to investigate the utility of venous phase delay assessment to evaluate the balloon occlusion test (BOT) of the internal carotid artery (ICA). A total of 38 patients who received BOT of the ICA were included in this retrospective study. Clinical examination and venous phase assessment were performed in all patients to evaluate their suitability for the evaluation of the BOT of the ICA. The venous phase delay assessment compared the venous phase of supratentorial and infratentorial structures between hemispheres. Venous phase delay was defined as the time lag for opacification of the first cortical vein between the occluded hemisphere and the hemisphere examined. The results of the clinical examination and the venous phase delay assessment were compared. In most patients negative on clinical examination, the venous phase delay was no more than $2 \mathrm{sec}$, while for most patients positive on clinical examination, the delay was $>2$ sec. All patients with a venous phase delay of $>4 \mathrm{sec}$ had a positive clinical result. The present results indicated that venous phase delay assessment is a reliable method for evaluating BOT of the ICA, and in those with a delay of $<2 \mathrm{sec}$, parent vessel occlusion of the ICA, which may be used as a pre-operative procedure prior to tumor resection for patients suffering from neck or skull-base tumors, was considered safe.
\end{abstract}

\section{Introduction}

When treating certain types of vascular disease, it is appropriate to perform parent vessel occlusion (PVO) of the diseased area. PVO may be used as a pre-operative procedure prior to tumor resection for patients suffering from neck or skull-base tumors (1,2). In such cases, internal carotid artery (ICA) endovascular occlusion is a necessary pre-operative maneuver. However,

Correspondence to: Professor En-Hua Xiao, Department of Radiology, The Second Xiangya Hospital of Central South University, 139 Renmin Zhong Lu, Changsha, Hunan 410011, P.R. China

E-mail: xiaoenhua64@csu.edu.cn

Key words: balloon occlusion test, internal carotid artery, venous phase delay, safety as there is a significant risk of ischemic events associated with ICA occlusion, patient selection is crucial. Thromboembolic and hemodynamic ischemia are the major complications associated with PVO (3). Therefore, it is crucial to identify patients that are likely to develop hemodynamic ischemia prior to PVO. A previous study demonstrated that temporary balloon occlusion of the ICA can be used to identify patients at risk for stroke during carotid artery sacrifice (4). The temporary balloon occlusion test (BOT) is useful in evaluating ischemic risk prior to permanent ICA occlusion (5). During the BOT, several methods may be used to assess the clinical tolerance of PVO, which include clinical examination assessment, venous phase delay assessment, stump pressure assessment, perfusion scanning assessment and neurophysiological monitoring (3). The clinical examination assessment is the most useful and broadly used method to determine whether a patient is able to tolerate PVO (6). There are several types of BOT assessment each with various advantages and disadvantages. For example, the CT perfusion method involves knowledge of image post-processing. This procedure also involves the transfer of the patient to the CT suite with a balloon catheter in the carotid artery, which may increase the risk of carotid artery injury (3). Venous phase delay assessment is a simple angiographic BOT criterion. To investigate the utility of venous phase delay assessment in evaluating the results from the BOT, the clinical examination assessment and the venous phase delay assessment during BOT of the ICA.

\section{Materials and methods}

Patients. A total of 38 patients without contraindications underwent a BOT of the ICA between January 2012 and July 2016 at The Second Xiangya Hospital of Central South University (Changsha, China). Clinical examinations and venous phase assessments were performed. Patients with a head and neck tumor or traumatic carotid cavernous fistulae were included in the study. The present study was approved by the Ethics Committee of the Second Xiangya Hospital of Central South University (Changsha, China). All patients provided informed consent.

BOT method. A 6-F femoral sheath was introduced into the femoral artery using the single-wall puncture technique. Cervical and cerebral angiograms were recorded with $4-\mathrm{F}$ diagnostic catheters in the anteroposterior and lateral projection, respectively. Each patient was given 70-100 units per 
$\mathrm{kg}$ of heparin prior to the angiogram. Subsequently, a 6-F guiding catheter was positioned in the common carotid artery. A non-detachable 4x15 mm silicon balloon catheter (cat. no. BCo415C; MicroVention Medical Technology, Co., Ltd., Hang Zhou, China) was positioned in the petrous ICA. After the diseased vessel was completely filled, the balloon was inflated for a total of $30 \mathrm{~min}$. During the $30 \mathrm{~min}$ procedure, a neurological examination was performed every $5 \mathrm{~min}$ (3). The patient was asked a series of questions to assess their motor, sensory, speech and memory capacity, as well as their analytical and/or calculation skills. If any neurological deficits were detected during balloon occlusion, the balloon catheter was immediately deflated. In this case, the clinical examination was considered positive. If no neurological deficit was detected by $30 \mathrm{~min}$, the result was negative.

Anteroposterior angiography was performed on the entire skull after the neurologic assessment. The venous phase delay assessment was performed by comparing the time difference for opacification of the first cortical vein between the territories of the examined hemisphere and the occluded hemisphere.

\section{Results}

Patient characteristics. The clinical characteristics of the 38 patients are listed in Table I. Of the 38 patients enrolled, $18(47 \%)$ were males and $20(53 \%)$ were females, average age 50 (16-72) years. There were 22 patients with carotid aneurysms, 12 with extensive cervical tumors and 4 with traumatic carotid cavernous fistulae. Fig. 1 displays a representative angiogram of a BOT of the right ICA in a 52-year-old male patient with a cervical tumor. A representative angiogram of a BOT of the right ICA in a 47-year-old male patient with a large cervical tumor is provided in Fig. 2.

Clinical and venous phase delay examination during BOT. A comparison of the results of the clinical examination and the venous phase assessment is provided in Table II. According to the neurological examination during BOT, 30 patients $(78.9 \%)$ were negative and $8(21.1 \%)$ were positive. Of the 30 negative patients, venous phase delay occurred within $2 \mathrm{sec}$ in 28 patients $(93.3 \%)$, and between 2 and $4 \mathrm{sec}$ in two patients (6.7\%). None of the patients with a negative result had a delay of $>4 \mathrm{sec}$. Of the eight patients with positive results, the venous phase delay was within $2 \mathrm{sec}$ for one patient $(12.5 \%)$, between 2 and $4 \mathrm{sec}$ for two patients (25\%) and $>4 \mathrm{sec}$ for five patients $(62.5 \%)$.

Venous phase delay during BOT may be utilized to determine suitability for $P V O$. Although the data were not sufficient from a statistical perspective, the results allow for the prediction that for most patients with a negative clinical examination the venous phase delay was $<2 \mathrm{sec}$. For the majority of patients with a positive clinical examination, the delay was $>2 \mathrm{sec}$. All patients with a venous phase delay of $>4 \mathrm{sec}$ had a positive clinical examination result.

\section{Discussion}

The treatment of complex vascular pathologies depends on the evolution of endovascular technology (3). For the treatment of certain vascular diseases, it is appropriate to perform PVO of the diseased area $(1,2)$. Certain conditions, including wide-neck giant aneurysms, pseudoaneurysms, traumatic vascular injuries, carotid blowout and arteriovenous fistulas, require PVO $(1,2)$. PVO may be used as a pre-operative treatment prior to tumor resection in patients suffering from neck or skull-base tumors (7). In the early 1970s, Serbinenko first reported on endovascular PVO with detachable balloons $(8,9)$. It is necessary to perform ICA endovascular occlusion as a pre-operative maneuver for the treatment of certain head and neck diseases (4).

Hemodynamic ischemia and thromboembolism are the major complications associated with PVO. Thromboembolic complications may be managed by anti-coagulation treatment (5). However, it is difficult to avoid immediate or delayed hemodynamic cerebral ischemia during carotid artery occlusion, even during uncomplicated procedures. Identification of patients that are likely to develop hemodynamic ischemia prior to PVO is crucial for preventing this type of complication (3).

A temporary BOT is useful for evaluating ischemic risk prior to permanent ICA occlusion. Several techniques may be used for the BOT. The primary purpose of the BOT is to assess the ability of the intracranial collateral circulation to maintain perfusion of the affected vascular territory during temporary occlusion of the major arterial supply. An interventionist assesses whether a patient is able to tolerate PVO by applying the BOT. A clinical BOT of the ICA contributes to lower post-occlusion morbidity (10,11). A study by Linskey et al (5), which included 516 patients, demonstrated that the use of an ICA BOT reduced the morbidity of permanent ICA occlusion from 26 to $13 \%$.

Several methods may be used to assess the clinical tolerance to PVO. These may include neurological examination assessment, venous phase delay assessment (6), stump pressure assessment (12), perfusion scanning assessment [including single-photon emission computed tomography (SPECT) (13), xenon CT perfusion (14), CT perfusion with an acetazolamide challenge (15)], as well as magnetic resonance (MR) perfusion (16) and neurophysiological monitoring (NPM) (17). Other methods include electroencephalography, somatosensory-evoked potentials and brain stem-evoked potentials (3), transcranial Doppler (TCD) ultrasonography (18) and induced hypotension (19). The aim of these techniques is to enhance the predictive value of a successful BOT. In certain cases, numerous assessment methods have been employed in conjunction with clinical examinations to determine in which patients $\mathrm{PVO}$ is safe and to thereby reduce the incidence of ischemic complications (20).

The neurological examination assessment is the most useful and widely applied method during BOT to determine whether a patient is able to tolerate PVO (21). When the BOT procedure is performed for clinical assessment, the patient is asked a series of questions to assess their motor, sensory, speech and memory capacity, as well as their analytical or calculation skills (3). Although the clinical assessment is the basis for all BOT paradigms, certain disadvantages exist. For instance, a clinical examination may increase the risk of thromboembolic complications, which may require a prolonged balloon inflation time. In addition, patients must be fully conscious and the occlusion procedure must be performed under local anesthesia to facilitate the clinical examination (3). 
Table I. Clinical characteristics of the 38 patients.

\begin{tabular}{llcrc}
\hline Sex & $\mathrm{n}$ & Carotid aneurysm & Extensive cervical tumor & Traumatic carotid cavernous fistulae \\
\hline Female & 20 & $13(65)$ & $5(25)$ & $2(10)$ \\
Male & 18 & $9(50)$ & $7(39)$ & $2(11)$ \\
Total & 38 & $22(58)$ & $12(31)$ & $4(11)$ \\
\hline
\end{tabular}

Values are expressed as $\mathrm{n}(\%)$.
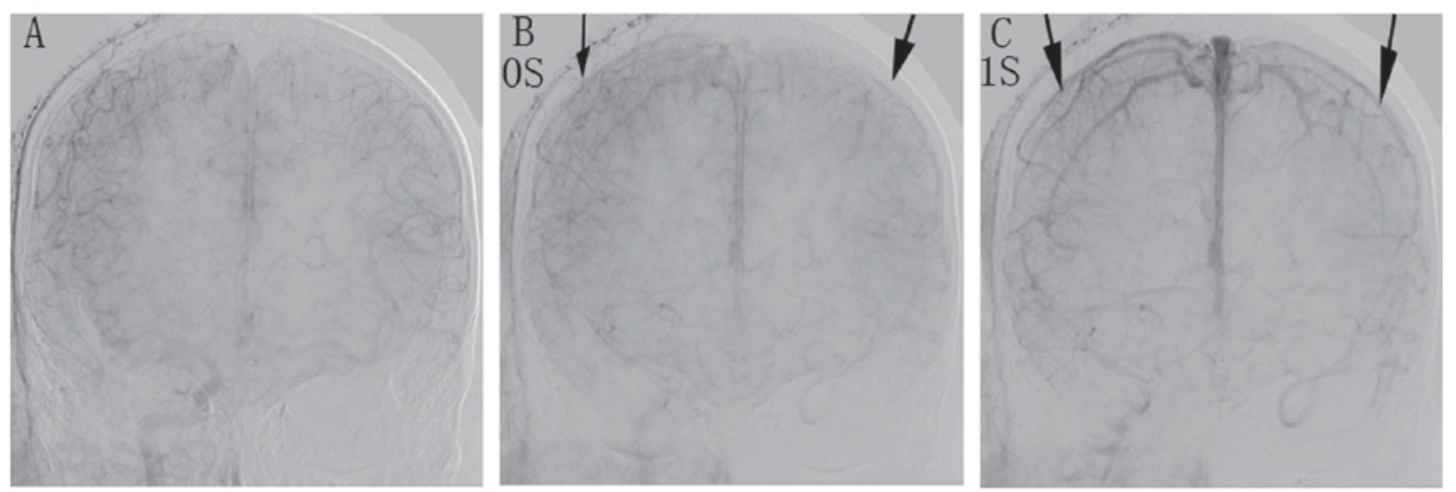

Figure 1. Angiogram displaying a BOT of the right internal carotid artery in a 52-year-old male patient with a cervical tumor. (A) Significant cross-filling of the Circle of Willis in the late arterial phase. (B) The early venous phase is displayed one sec after the first image; defined as 0S. Cortical vein opacification is observed in the left and right hemispheres (black arrows). (C) One sec later (1S), the venous phase synchrony is more evident in the left and right hemispheres (black arrows). This patient represents an example of perfect symmetry of venous phase delay during a BOT. BOT, balloon occlusion test; 0S, 0 sec; $1 \mathrm{~S}$, one sec.
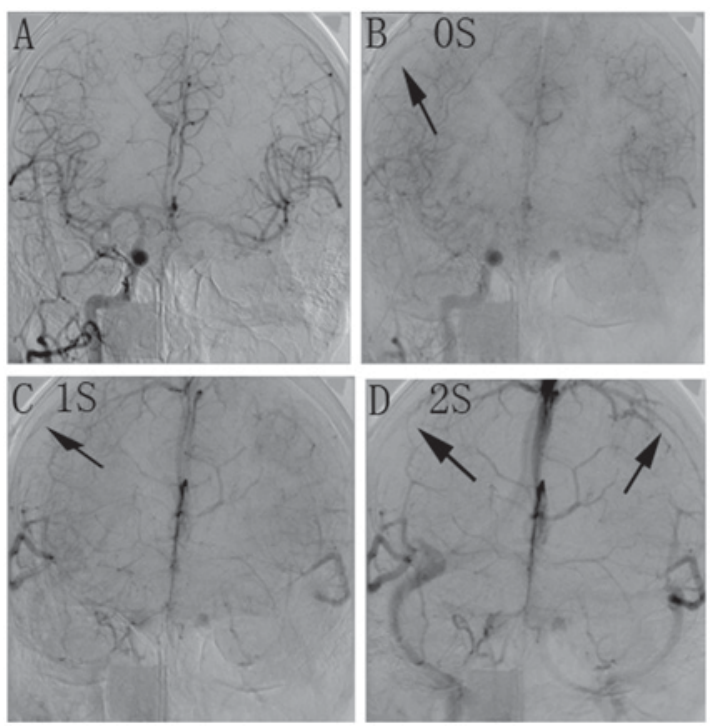

Figure 2. Angiogram displaying a BOT of the right internal carotid artery in a 47-year-old male patient with a cervical tumor. (A) Significant cross-filling of the Circle of Willis in the late arterial phase. (B) The beginning of the venous phase on the right hemisphere (black arrow) and certain cortical arteries in the left hemisphere are visible one sec after the first image; defined as 0S. (C) One second later (1S), the cortical arteries on the left hemisphere and veins on the right hemisphere (black arrows) are more visible. (D) One second later (i.e., the venous phase at $2 \mathrm{~S}$ ), the veins were filled in the left and right hemispheres (black arrows). This patient had a $2 \mathrm{sec}$ venous drainage delay. BOT, balloon occlusion test; $0 \mathrm{~S}, 0 \mathrm{sec} ; 1 \mathrm{~S}$, one sec; $2 \mathrm{~S}$, two sec.

For most types of BOT assessment, certain challenges prevail, as the systemic pressure is affected by several factors.
General anesthesia, patient mood and even environmental conditions may affect stump pressure assessment (20). In addition, although previous studies have identified a significant correlation between stump pressure and cerebral perfusion (22), the usefulness of stump pressure assessment remains controversial, as other studies did not identify a correlation with cerebral perfusion (23). If perfusion scanning assessment is performed, the patient must be transferred to a CT, MRI or SPECT scanning room. The balloon is placed in the ICA during transportation, which may increase the risk of carotid artery injury (13-15). The disadvantage of NPM is that the result is not reliable due to the high false-positive and -negative rates (17). Although TCD ultrasonography has the advantage of being non-invasive, it does not provide a consistently stable result (18). The correlation between cerebral blood flow and mean middle cerebral artery velocity is not linear, as the mean velocity may be affected by the hematocrit, viscosity and vessel caliber (14). A previous study indicated that the induced hypotension assessment was not superior to the traditional BOT (19). Dare et al (19) suggested that the false-negative rate of the induced hypotension assessment may be increased due to the direct vasodilator effect of nitroprusside on the cerebral circulation.

The venous phase delay assessment is based on the assumption that an adequate intracranial collateral circulation exists to confirm the symmetry of the two hemispheres when one ICA is occluded during a BOT (6). Venous phase delay is a simple angiographic BOT assessment performed under general anesthesia removing the need for any further neurological examination. This type of evaluation is rapid and 
Table II. Results of the clinical examination and venous phase assessment.

\begin{tabular}{lrccc}
\hline & & \multicolumn{3}{c}{ Venous phase delay (sec) } \\
\cline { 3 - 5 } Clinical examination & $\mathrm{n}$ & $<2$ & $2-4$ & $>4$ \\
\hline Negative & 30 & $28(93)$ & $2(7)$ & $0(0)$ \\
Positive & 8 & $1(12.5)$ & $2(25)$ & $5(62.5)$ \\
Total & 38 & $29(76)$ & $4(11)$ & $5(13)$
\end{tabular}

Values are expressed as n (\%).

straightforward (6). A reduced balloon inflation time in the ICA thus indicates a reduced risk of thrombus formation associated with an occluded ICA (3). Even though venous phase delay has several advantages and has recently attracted extensive attention, neurological examination remains the most commonly used method to assess a patient's ability to tolerate PVO (21). Neurological examination is straightforward, practicable and does not require any specialized equipment. Accordingly, in the present retrospective study, the results of the venous phase delay assessment were compared with those of a neurological examination during a BOT of the ICA to investigate a preliminary comparison between the two types of assessment.

In the present study, for most patients with a negative neurological examination, the venous phase delay was $<2 \mathrm{sec}$. Therefore, patients with a venous phase delay $<2 \mathrm{sec}$ were identified as having the ability to tolerate PVO. This result was consistent with that of a study by Abud et al (6), which demonstrated that patients with a venous phase delay $<3 \mathrm{sec}$ were identified as having the ability to tolerate carotid occlusion without developing neurologic deficit. For most patients with a positive neurological examination, the venous phase delay was $>2 \mathrm{sec}$, and all patients with a venous phase delay of $>4 \mathrm{sec}$ had a positive neurological examination. Therefore, a venous phase delay of $>2 \mathrm{sec}$, which is associated with a positive neurological examination during BOT, may be used to indicate patients which cannot tolerate PVO. Furthermore, patients with a venous phase delay of $>4$ sec will definitely not be able to tolerate PVO. Accordingly, if the venous phase delay is 2-4 sec, the BOT result requires to be determined by additional factors along with the venous phase delay.

In conclusion, according to the present retrospective study and previous studies, venous phase delay assessment is a reliable method for evaluating a BOT of the ICA. In addition, a delay $<2 \mathrm{sec}$ should be considered to indicate that PVO of the ICA is safe.

\section{Acknowledgements}

Not applicable.

\section{Funding}

The present study was supported by grants from the National Natural Science Foundation of China (grant nos. 81601471 and 81571784); the Scientific and Technological Support
Project in the Field of Social Development of Hunan Province (grant no. 2015SF2020-4) and the Project of Development and Reform Commission of Hunan Province (grant no. Xiangcai enterprise means [2015] 83).

\section{Availability of data and materials}

All datasets used and/or analyzed during the current study are available from the corresponding author on reasonable request.

\section{Authors' contributions}

EX and HZ designed the study. ZC and LH performed the examinations and analyzed the data. ZC and $\mathrm{LH}$ prepared the manuscript. EX and $\mathrm{HZ}$ read and approved the final manuscript.

\section{Ethical approval and consent to participate}

The present study was approved by the Ethics Committee of the Second Xiangya Hospital of Central South University (Changsha, China). All patients provided informed consent.

\section{Patient consent for publication}

Not applicable.

\section{Competing interests}

The authors declare that they have no competing interests.

\section{References}

1. Snelling BM, Sur S, Shah SS, Wolfson RI, Ambekar S, Yavagal DR, Elhammady MS and Peterson EC: Venous phase timing does not predict SPECT results during balloon test occlusion of the internal carotid artery. World Neurosurg 102: 229-234, 2017.

2. Bavinzski G, Killer M, Ferraz-Leite H, Gruber A, Gross CE and Richling B: Endovascular therapy of idiopathic cavernous aneurysms over 11 years. AJNR Am J Neuroradiol 19: 559-565, 1998.

3. Elias AE, Chaudhary N, Pandey AS and Gemmete JJ: Intracranial endovascular balloon test occlusion: Indications, methods, and predictive value. Neuroimaging Clin N Am 23: 695-702, 2013.

4. Mathis JM, Barr JD, Jungreis CA, Yonas H, Sekhar LN, Vincent D, Pentheny SL and Horton JA: Temporary balloon test occlusion of the internal carotid artery: Experience in 500 cases. AJNR Am J Neuroradiol 16: 749-754, 1995.

5. Linskey ME, Jungreis CA, Yonas H, Hirsch WL Jr, Sekhar LN, Horton JA and Janosky JE: Stroke risk after abrupt internal carotid artery sacrifice: Accuracy of preoperative assessment with balloon test occlusion and stable xenon-enhanced CT. AJNR Am J Neuroradiol 15: 829-843, 1994.

6. Abud DG, Spelle L, Piotin M, Mounayer C, Vanzin JR and Moret J: Venous phase timing during balloon test occlusion as a criterion for permanent internal carotid artery sacrifice. AJNR Am J Neuroradiol 26: 2602-2609, 2005.

7. Hertel A, Görling S, Schwager K and Hofmann E: Angiography and cerebral perfusion scintigraphy in balloon test occlusion of carotid artery in head and neck tumors. Rofo 184: 214-219, 2012.

8. Serbinenko F: Catheterization and occlusion of major cerebral vessels and prospects for the development of vascular neurosurgery. Vopr Neirokhir 35: 17-27, 1971 (In Russian).

9. Serbinenko FA: Balloon catheterization and occlusion of major cerebral vessels. J Neurosurg 41: 125-145, 1974.

10. Detre JA, Samuels OB, Alsop DC, Gonzalez-At JB, Kasner SE and Raps EC: Noninvasive magnetic resonance imaging evaluation of cerebral blood flow with acetazolamide challenge in patients with cerebrovascular stenosis. J Magn Reson Imaging 10: 870-875, 1999. 
11. Kuwabara Y, Ichiya Y, Sasaki M, Akashi Y, Yoshida T, Fukumura T and Masuda K: A comparison of the cerebrovascular responses to $\mathrm{CO} 2$ and Diamox in patients with unilateral occlusive cerebral arteries: A H2(15)O PET study. Kaku Igaku 32: 569-577, 1995 (In Japanese).

12. Morishima H, Kurata A, Miyasaka Y, Fujii K and Kan S: Efficacy of the stump pressure ratio as a guide to the safety of permanent occlusion of the internal carotid artery. Neurol Res 20: 732-736, 1998.

13. Tansavatdi K, Dublin AB, Donald PJ and Dahlin B: Combined balloon test occlusion and SPECT analysis for carotid sacrifice: Angiographic predictors for success or failure?. J Neurol Surg B Skull Base 76: 249-251, 2015.

14. Kofke WA, Brauer P, Policare R, Penthany S, Barker D and Horton J: Middle cerebral artery blood flow velocity and stable xenon-enhanced computed tomographic blood flow during balloon test occlusion of the internal carotid artery. Stroke 26: 1603-1606, 1995.

15. Jain R, Hoeffner EG, Deveikis JP, Harrigan MR, Thompson BG and Mukherji SK: Carotid perfusion CT with balloon occlusion and acetazolamide challenge test: Feasibility. Radiology 231: 906-913, 2004.

16. Ma J, Mehrkens JH, Holtmannspoetter M, Linke R, Schmid-Elsaesser R, Steiger HJ, Brueckmann $\mathrm{H}$ and Bruening R: Perfusion MRI before and after acetazolamide administration for assessment of cerebrovascular reserve capacity in patients with symptomatic internal carotid artery (ICA) occlusion: Comparison with 99mTc-ECD SPECT. Neuroradiology 49: 317-326, 2007.

17. Liu AY, Lopez JR, Do HM, Steinberg GK, Cockroft K and Marks MP: Neurophysiological monitoring in the endovascular therapy of aneurysms. AJNR Am J Neuroradiol 24: 1520-1527, 2003.
18. Eckert B, Thie A, Carvajal M, Groden C and Zeumer $\mathrm{H}$ : Predicting hemodynamic ischemia by transcranial Doppler monitoring during therapeutic balloon occlusion of the internal carotid artery. AJNR Am J Neuroradiol 19: 577-582, 1998.

19. Dare AO, Chaloupka JC, Putman CM, Fayad PB and Awad IA Failure of the hypotensive provocative test during temporary balloon test occlusion of the internal carotid artery to predict delayed hemodynamic ischemia after therapeutic carotid occlusion. Surg Neurol 50: 147-156, 1998.

20. Wang AY, Chen CC, Lai HY and Lee ST: Balloon test occlusion of the internal carotid artery with stump pressure ratio and venous phase delay technique. J Stroke Cerebrovasc Dis 22: e533-e540, 2013

21. Asai K, Imamura H, Mineharu Y, Tani S, Adachi H, Narumi O, Sato S, Sakai C and Sakai N: X-ray angiography perfusion analysis for the balloon occlusion test of the internal carotid artery. J Stroke Cerebrovasc Dis 24: 1506-1512, 2015.

22. Tomura N, Omachi K, Takahashi S, Sakuma I, Otani T, Watarai J, Ishikawa K, Kinouchi $\mathrm{H}$ and Mizoi K: Comparison of technetium Tc $99 \mathrm{~m}$ hexamethylpropyleneamine oxime single-photon emission tomograph with stump pressure during the balloon occlusion test of the internal carotid artery. AJNR Am J Neuroradiol 26: 1937-1942, 2005

23. Barker DW, Jungreis CA, Horton JA, Pentheny S and Lemley T: Balloon test occlusion of the internal carotid artery: Change in stump pressure over 15 minutes and its correlation with xenon CT cerebral blood flow. AJNR Am J Neuroradiol 14: 587-590, 1993. 